\title{
The Effects of Time Varying Intravascular Signal Intensity and k- Space Acquisition Order on Three- Dimensional MR Angiography Image Quality
}

\author{
Jeffrey H. Maki, MD, PhD • Martin R. Prince, MD, PhD • Frank J. Londy, RT(R) • Thomas L. Chenevert, PhD
}

\begin{abstract}
The optimum infusion timing and $\mathbf{k}$-space ordering for obtaining gadolinium-enhanced three-dimensional MR angiograms was determined through computer modeling using temporal contrast characteristics obtained from patient gadolinium infusion data. The effects of bolus timing were evaluated by varying the relationship between peak intravascular gadolinium concentration and the time at which the center of $k$ space was acquired $\left(t_{\mathrm{ck}}\right)$ for sequential and centric acquisition techniques. Flow phantom experiments were performed to validate the theoretical computations.

Gadolinium concentration at the time of central $\mathbf{k}$ space acquisition determines intravascular signal intensity. Artifacts, including vessel broadening and edge ringing, depend on the order in which $\mathrm{k}$ space is collected and on how rapidly the gadolinium concentration changes. Artifacts are greatest when the center of $k$ space is acquired before the intravascular gadolinlum peak. Application of the optimal infusion timing results in preferential arterial enhancement with a minimum of artifacts in patients undergoing MR angiography.
\end{abstract}

Index terms: Magnetic resonance - Magnetic rcsonance angiography $\cdot$ Contrast enhancement $\cdot$ Centric $\cdot$ Computer modeling

JMRI 1996; 6:642-651

Abbreviations: DFT $=$ discrete Fourler transform, Gd-MRA $=$ gadoliniumenhanced MR angiography, IV = intravenous, IVC = inferior vena cava, MIP $=$ maximum intensity projections.

From the Department of Radiology, University of Michigan, Ann Arbor, Michigan. Recelved December 5, 1995; accepted April 1, 1996. Address reprint requests to J.H.M., Department of Radiology, University of Michigan, 1500 East Medical Center Drive, Ann Arbor, MI 48109-0030.
3D GRADIENT ECHO MR IMAGING during the arterial phase of a gadolinium infusion demonstrates arteries distinct from veins and background tissues (1-4). Compared to time-of-flight or phase-contrast angiography, flow and saturation effects are markedly decreased $(3,5-$ 8). Anecdotal reports indicate that image quality is optimized when the contrast infusion is timed to maximize arterial gadolinium concentration during the acquisition of the central portion of $\mathbf{k}$ space. In our experience, variations in infusion timing can dramatically affect the relative degree of arterial and venous enhancement. In addition, the changing intravascular gadolinium concentration over the relatively long data-acquisition period produces interesting contrast effects and artifacts.

This study evaluates the impact of time-varying intravascular contrast and $\mathrm{k}$-space ordering on gadoliniumenhanced MR angiography (Gd-MRA). In particular, the relationship between the center of $k$ space and the time course of intravascular contrast enhancement is explored to determine an optimal injection timing and $\mathbf{k}$-space ordering strategy.

\section{- THEORY}

\section{Data Acquisition Technique}

Of the many techniques of ordering $\mathrm{k}$-space acquisition, two are investigated in this work: sequential and centric $(9,10)$. In sequential Fourier spin-warp imaging, the most negative spatial frequency component is collected first, with each subsequent data point advancing through the zero spatial frequency (DC) component up to the most positive spatial frequency component. With centric acquisition, the zero spatial frequency (DC) component is collected first, followed by alternating positive and negative higher and higher spatial frequency components. These two methods are illustrated in Figure 1.

\section{Time-Varying Intravascular Signal}

Gd-MRA is performed using a three-dimensional gradient-echo acquisition $(1-6,8,11)$. Three-dimensional computer modeling of such a sequence is computationally time intensive. Therefore, several simplifications can be made to reduce the problem to a single dimension. 


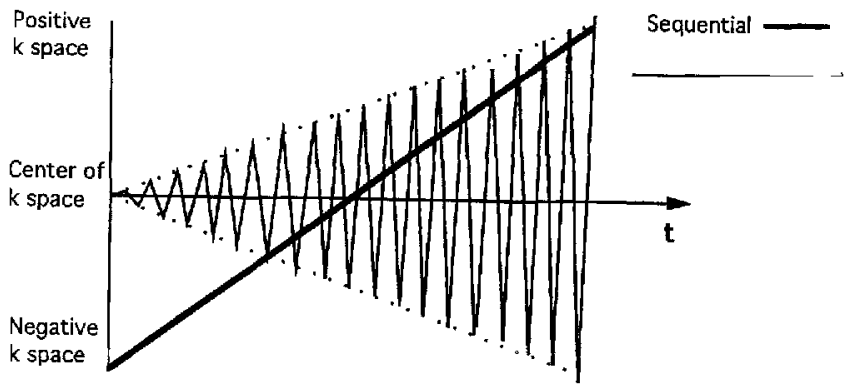

Figure 1. Time order of sequential and centric data acquisition.

This makes the computation less cumbersome but still allows for meaningful analysis and extrapolation of the results.

Consider a volume of tissue containing a vessel with time-varying gadolinium concentration such that signal from any point can be described as $f(x, y, z, t)$. Because three-dimensional MR collects data describing the $3 \mathrm{D}$ discrete Fourier transform (DFT) of the actual object, we can say

$$
\hat{F}=\sum_{n_{x}=1}^{R} e^{-i n_{x} k_{x}} \sum_{n_{y}=1}^{N} e^{i r_{y}, k_{y}} \sum_{n_{z}-1}^{S} e^{-i n_{z} k_{z}} f\left(n_{x}, n_{y}, n_{z}, t\right)
$$

where $\hat{F}=\hat{F}\left(k_{x}, k_{y}, k_{z}, t\right)$ is the DFT of $f(x, y, z, t), R$ is the number of readout-encoding steps, $N$ is the number of phase-encoding steps for each slice, and $S$ is the number of slices.

Now assume that readout time is instantaneous (a reasonable assumption because readout time is on the order of milliseconds). Object signal intensity is then effectively constant over the readout time, and hence, the spatial features along the readout axis will be preserved faithfully. If we now choose a special geometry such that there is no $z$ dependence (see Fig. 2), the Fourier transform in the $z$ direction approximates a delta function. Under this set of circumstances, the problem can be simplified to a single dimension. This is demonstrated in Figure 2, in which a single slice in $y$ and $z$ is selected, from which a line profile (along $y$ ) is generated. Anatomically, this corresponds to axial imaging of a purely longitudinal aorta or sagittal imaging of a purely transverse renal artery.

If the vessel spatial and temporal intensity functions are factored as $f\left(n_{y}, t\right)=g\left(n_{y}\right) d(t)$, the Fourier transform of the resultant line profile can be written as:

$$
\begin{aligned}
\hat{F}\left(k_{y}, t\right) & =c(t) \sum_{n_{y}=1}^{N} e^{-i n_{y} k_{y}} g\left(n_{y}\right) \\
& =c(t) \hat{G}\left(k_{y}\right)
\end{aligned}
$$

where $\hat{G}\left(k_{\mathrm{y}}\right)$ is the Fourier transform of $g\left(n_{y}\right)$. The function $c(t)$ can be thought of as a "filter" applied to $\hat{G}\left(k_{y}\right)$. Depending on the order in which $\hat{G}\left(k_{y}\right)$ is collected (see Fig. 1), a relationship between time $t$ and phase-encoding step $k_{y}$ can be determined. This allows us to rewrite $c(t)$ as a filter function that we term $\kappa\left(k_{y}\right)$, and thus Equation [2] can be rewritten as $\hat{F}\left(k_{y}\right)=\kappa\left(k_{y}\right) \hat{G}\left(k_{y}\right)$.

If data are acquired in the conventional "sequential" order with $k_{y}$ as the outermost or "slow" phase-encoding variable, a linear relationship exists between $t$ and $k_{y}$ such that $t=\mathrm{TR}^{*} S^{*} k_{y}$ (single NEX, TR $=$ repetition time, $\mathrm{S}=$ number of slice phase-encoding steps), and hence $\kappa\left(k_{y}\right)=c\left(\mathrm{TR}^{*} S^{*} k_{y}\right)$. Alternatively, data may be acquired in a "centric" order, in which case the filter function $\kappa\left(k_{u}\right)$ is a rearranged version of $c\left(\mathrm{TR}^{*} \mathrm{~S}^{*} k_{y}\right)$ corresponding in time to the alternating nature of centric $\mathbf{k}$-space acquisition,

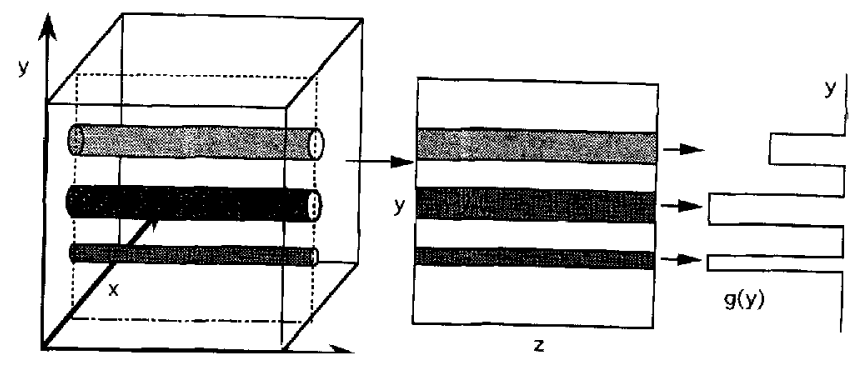

Figure 2. Three-dimensional geometry used for computer simulations. Vessels are oriented in the $z$ direction such that there is no $z$ dependence. A single slice of the three-dimensional data set is selected from which a one-dimensional line profile is generated. Darker shading corresponds to increased signal.

as demonstrated in Figure 3. This is further discussed under Methods.

Summarizing the preceding discussion, a one-dimensional projection of the output MR image lalong the $y$ axis of any $y-z$ plane slice in our three-dimensional volume having this special geometry) can be calculated as follows. Take the one-dimensional Fourier transform of the function $g(y)$ (describing the "true" geometry) to obtain $\hat{G}\left(k_{y}\right)$. Point by point multiply $\bar{G}\left(k_{y}\right)$ with the filter function $\kappa\left(k_{y}\right)$, which represents an acquisition-dependent (sequential versus centric) reordering of the enhancement curve $c(t)$. Finally, take the inverse Fourier transform of the product.

This model can be generalized to include $M$ vessels with the geometry $g_{m}(y)$, each with different time-varying enhancement functions $c_{m}(t)$. In this case, the net result is:

$$
\hat{F}\left(k_{y}\right)=\sum_{m=1}^{M} \kappa_{m}\left(k_{y}\right) \hat{G}_{m}\left(k_{y}\right)
$$

where $\hat{G}_{r n}\left(k_{y}\right)$ is the Fourier transform of $g_{m}(y)$.

Although the preceding theoretical analysis is mathematically accurate, the assumption of no $z$ dependence in the imaged volume is somewhat unrealistic when applied to most vascular anatomy. Nonetheless, the analysis is applicable to more complicated geometries, provided that the time course of data collection along the innermost or "fast" phase-encoding direction $\left(k_{z}\right)$ is relatively short compared to the time course of intravascular enhancement. Typical Gd-MRA imaging parameters might be a TR of $11 \mathrm{msec}, 32$ slices, and 128 phase-encoding steps. Under these circumstances, the time course of data collection along the "slow" axis is .35 sec and total imaging time is $45 \mathrm{sec}$. Based on the vessel enhancement data presented in Figure 4, except during the period of extremely abrupt aortic enhancement, intravascular signal changes during the $.35 \mathrm{sec}$ "fast" phase-encoding duration are relatively small, at least compared to the overall change during the 45-second "slow"-encoding duration. Thus, at least to first order approximation, time-varying intravascular signal during the "fast"encoding direction can be ignored, making the model presented here generalizable to more complicated anatomy.

\section{- METHODS}

\section{Computational Model Calculations}

Vessel enhancement data were based on the work of Strouse et al, who evaluated signal intensity versus time in the aorta and inferior vena cava (IVC) after the admin- 

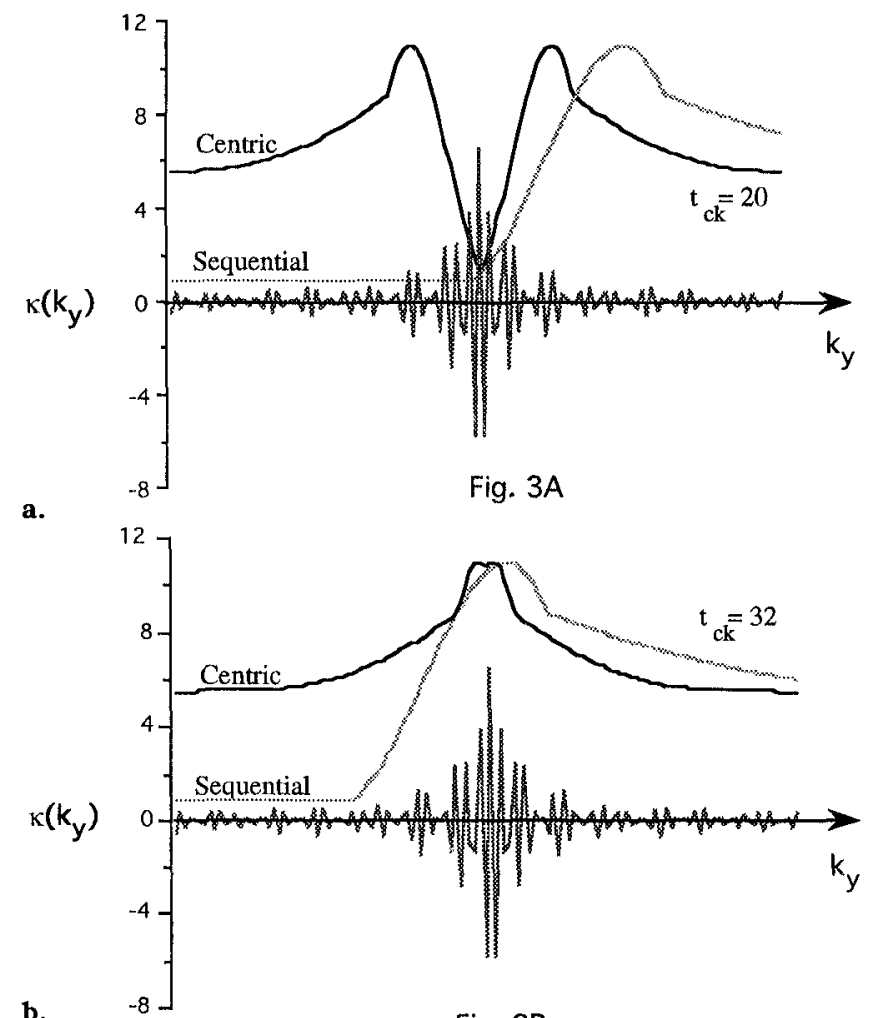

Fig. 3B

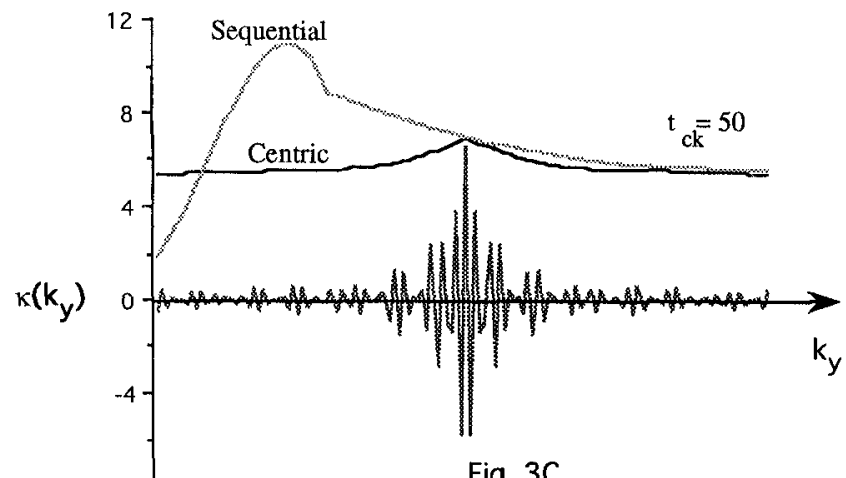

c.

Figure 3. Sequential and centric filter functions $\kappa\left(k_{y}\right)$ superimposed on one-dimensional k-space data for: (a) $t_{\mathrm{ccc}}=20 \mathrm{sec}-$ onds, (b) $t_{c k}=32$ seconds, and (c) $t_{c k}=50$ seconds. The $x$ axis is phase-encoding number ( 1 through $N$ ), the $y$ axis represents both $\kappa\left(k_{t}\right)$ and relative $\mathrm{k}$-space data amplitude, and $t$ is the time at which the center element of $\mathrm{k}$ space is acquired. $k\left(k_{\mathrm{y}}\right)$ is obtained from Figure 4.

istration of intravenous gadolinium (12). The contrastversus-time curves used to model aorta and IVC signal are shown in Figure 4 . This data set was obtained by averaging the results from four patients after a 10-second intravenous (IV) bolus of . $1 \mathrm{mmol} / \mathrm{kg}$ of gadopentetate dimeglumine.

The effects of time-varying intravascular enhancement during MRA image acquisition were quantified through one-dimensional modeling as described in the Theory section. From the discussion leading up to Equation [3], this process consists of filtering the $\mathrm{k}$-space data for each vessel by a modulation function $\kappa\left(k_{y}\right)$, which is related to the time-varying enhancement function $c(t)$. For either imaging technique, $N$ (number of phase-encoding steps) points of $\mathrm{k}$ space are collected linearly in time at integer

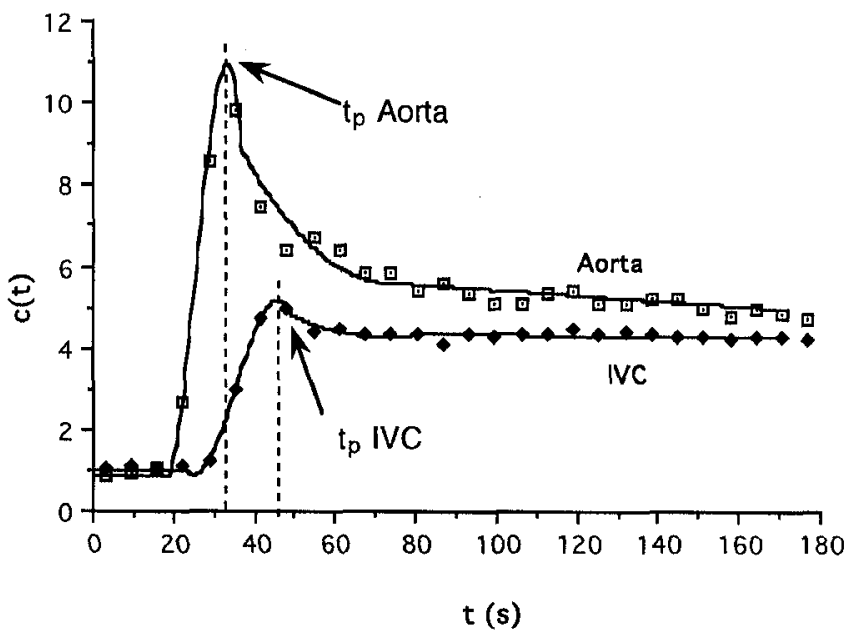

Figure 4. Human aorta and IVC enhancement curves $c(t)$ measured in vivo (11) and used in the modeling simulations. Time $t$ represents the point of maximal enhancement.

multiples of TR* $S$ (where $S$ is the number of slices), and hence the first acquired element of $\mathrm{k}$ space is multiplied by $c\left(\mathrm{TR}^{*} S\right)$, the second by $c\left(2 \mathrm{TR}^{*} S\right)$, the third by $c\left(3 \mathrm{TR}^{*} S\right)$, etc. For sequential acquisition, $\kappa\left(k_{t}\right)$ is simply $c\left(T^{*} S^{*} k_{t}\right)$. For centric acquisition, it is somewhat more complicated, as $\kappa\left(k_{y}\right)$ is a reordered version of $c\left(\mathrm{TR}^{*} S^{*} k_{u}\right)$ so that the first element of $\mathrm{k}$ space is multiplied by $c(N / 2 * T R * S)$, the second by $c\left[[N / 2+1]^{*} T^{*} S\right)$, the third by $c([N / 2-$ $\left.1]^{*} T R * S\right)$, the fourth by $c([N / 2+2] * T R * S)$, etc. A technique that helps better visualize this concept is to display the echo as if it were collected in a sequential manner and superimpose it with the appropriate $\kappa\left(k_{y}\right)$. This is shown in Figures 3a through 3c, with the bolus timing varied so that peak enhancement occurs before, at, and after acquisition of the center of $\mathrm{k}$ space $\left(t_{c k}\right)$.

Equation [3] was implemented on a MacIntosh computer using MATLAB software (MathWorks, Inc., Natick, MA). The modeled volume consisted of a series of different-sized tubular "vessels" running in the $z$ direction to satisfy the assumptions made in the Theory section (see Fig. 1). Each "vessel" was allowed to have its own timevarying contrast function so that both "arterial" and "venous" structures were modeled simultaneously. Timevarying contrast data were input as a mathematical function of time, as shown in Figure 4. Simulations were performed where the center of $\mathrm{k}$ space $\left(t_{c k}\right)$ occurred at 20,32 , and 50 seconds. The geometry modeled (Fig. 5) consisted of two arterial vessels, one large and one small ( 2 and 12 pixels out of 128 , signal enhancement modeled as aorta), and a single large venous vessel (12 pixels, signal enhancement modeled as IVC). The modeling was performed using a TR of 14 msec, 32 slices, and 128 phase-encoding steps corresponding to a total imaging time of approximately 57 seconds.

\section{Phantom Model}

To validate the theoretical modeling, the computational experiment was duplicated in a flow phantom. The phantom was constructed using four rigid Lucite tubes connected in series, aligned in a horizontal plane, and oriented with their longitudinal axes down the bore of the magnet. Each tube was $.96 \mathrm{~cm}$ in diameter and approximately $80 \mathrm{~cm}$ in length. Approximately $40 \mathrm{~cm}$ of Tygon tubing was used to connect the tubes, as well as for inflow and exhaust. Tap water was regulated to flow through the apparatus via a constant head gravity feed 
Sequential

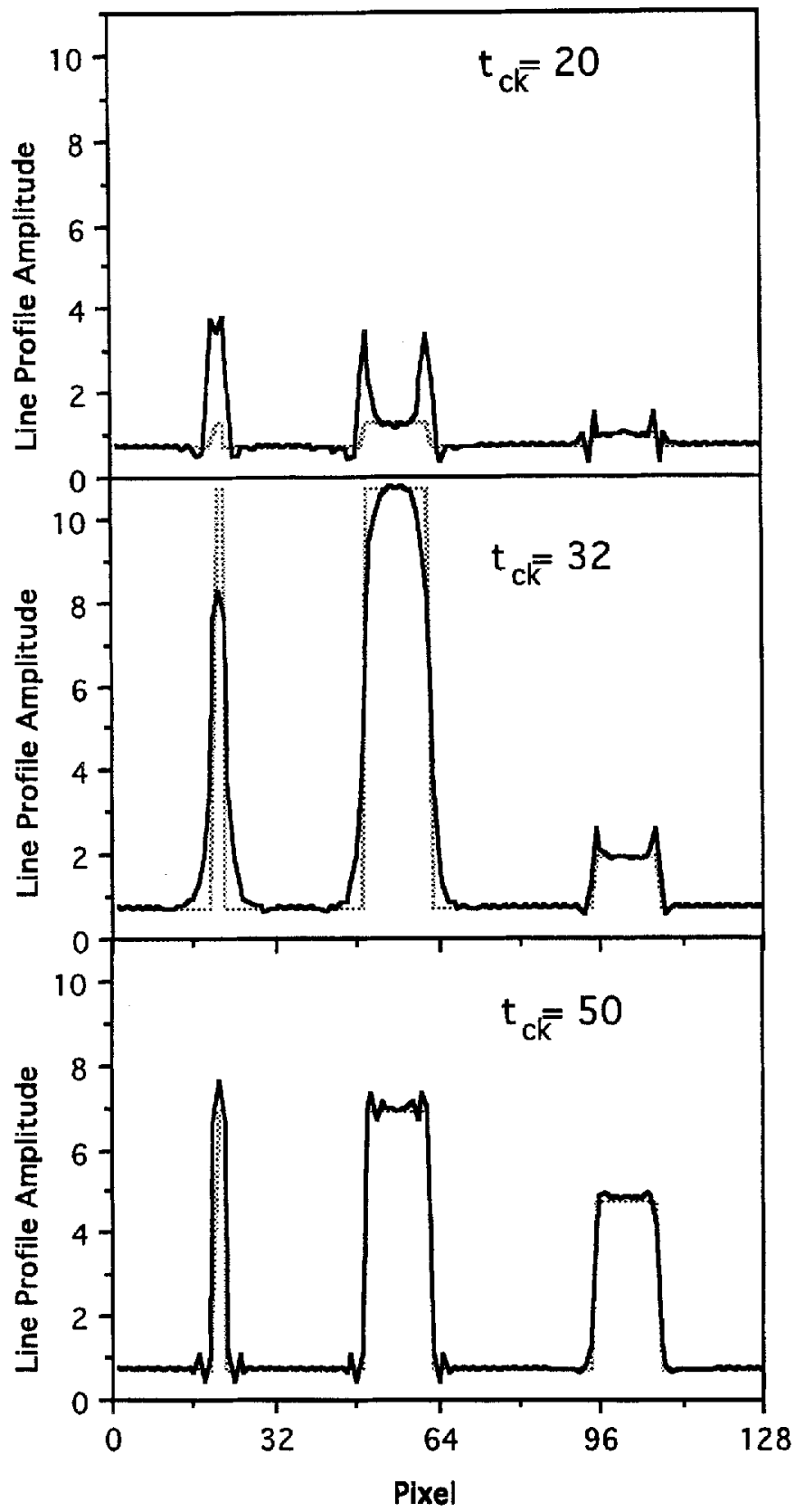

Centric

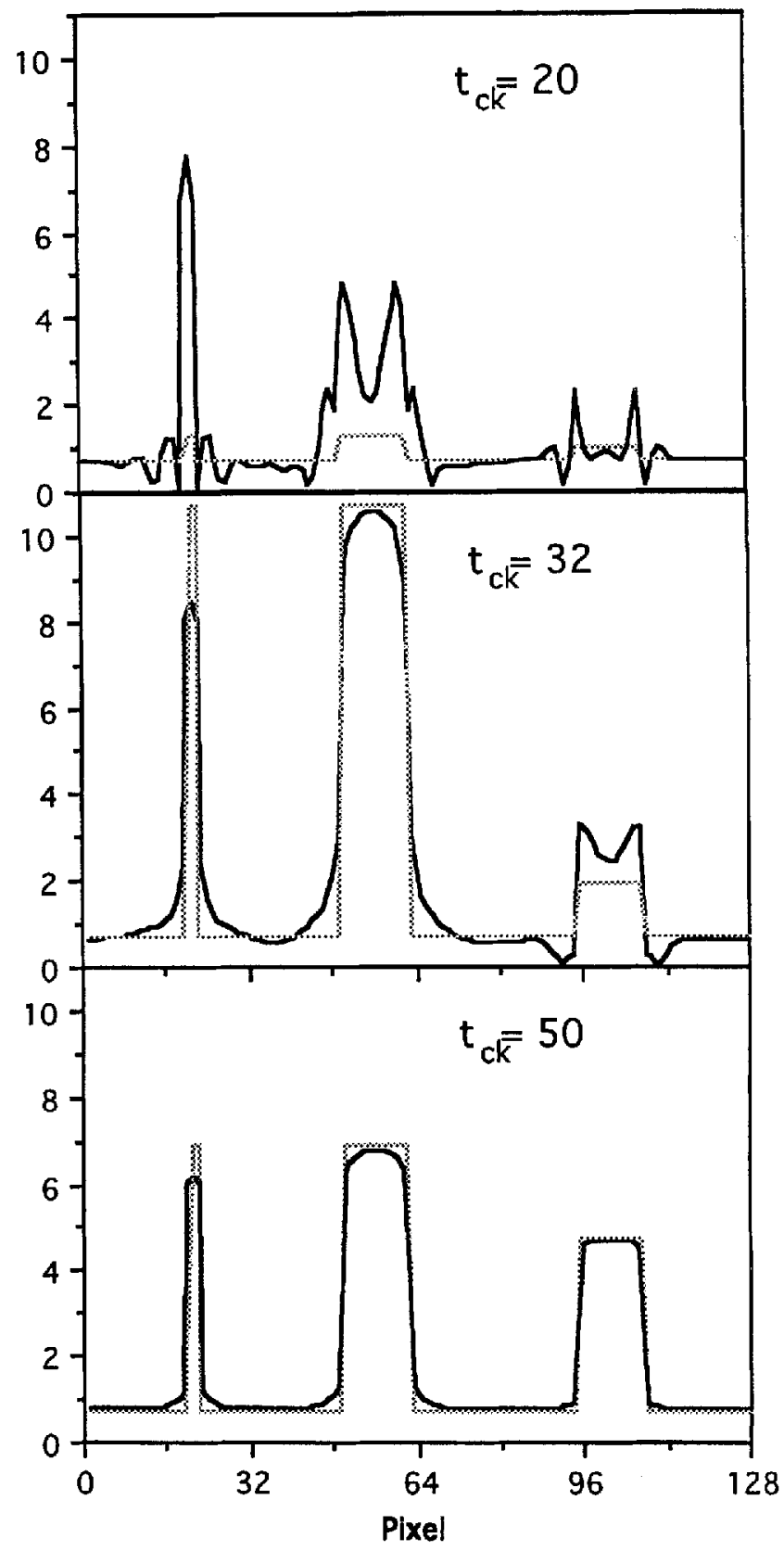

Figure 5. Computer-modeled sequential and centric line profiles for $t_{c k}=20,32$, and 50 seconds. Modeling based on the enhancement data $c(t)$ from Figure 4 . Dotted lines represent the true instantaneous "vessel" line profile at $t_{c k}$, ie, signal intensity $=c\left(l_{c k}\right)$.

tank at approximately $130 \mathrm{ml} / \mathrm{min}$. This provided a steady, reproducible average in tube velocity of $3 \mathrm{~cm} / \mathrm{sec}$. This slow velocity minimized inflow artifacts. To achieve an enhancement effect similar to that seen physiologically, a 1-ml bolus of gadopentetate dimeglumine was followed by a $.42-\mathrm{ml} / \mathrm{min}$ constant infusion. This was injected into the tubing approximately 2 meters upstream from the first tube using a Lifecare $5000 \mathrm{IV}$ pump (Abbott Laboratories, Abbott Park, IL). To adequately load the coil, multiple saline bags and a plastic bottle of tap water were placed in the head coil adjacent to the phantom.

Experiments were performed on a GE Signa 1.5-T super-conducting MR system (General Electric Medical Systems, Milwaukee, WI) running 5.4 software. Before three-dimensional experiments, the temporal enhancement properties of the phantom were evaluated at higher temporal resolution using serial thick-slice two-dimensional acquisitions. Using the head coil, a coronal thickslice dynamic two-dimensional gradient-echo sequence (fast spoiled gradient recalled echo; slice thickness, 20 $\mathrm{mm}$; FOV, $20 \mathrm{~cm}$; TR, $7.8 \mathrm{msec}$; TE, $2.5 \mathrm{msec}$; flip angle, $45^{\circ} ; \mathrm{NEX}=8$; matrix $256 \times 128$ ) was performed, yielding 20 sequential images at 8.02 -second intervals. The procedure was repeated once to ensure reproducibility, and regions of interest were measured in the central portion of each tube.

Three-dimensional MRA phantom images were then obtained using a three-dimensional spoiled gradientecho sequence (32 slices; slice thickness, 2 mm; FOV, 20 $\mathrm{cm}$; TR, $10.9 \mathrm{msec}$;E, $2.3 \mathrm{msec}$; flip angle, $45^{\circ}$; NEX = 1 ; matrix $256 \times 128$ ). This sequence was modified by one of the authors to acquire data in either the sequential or 


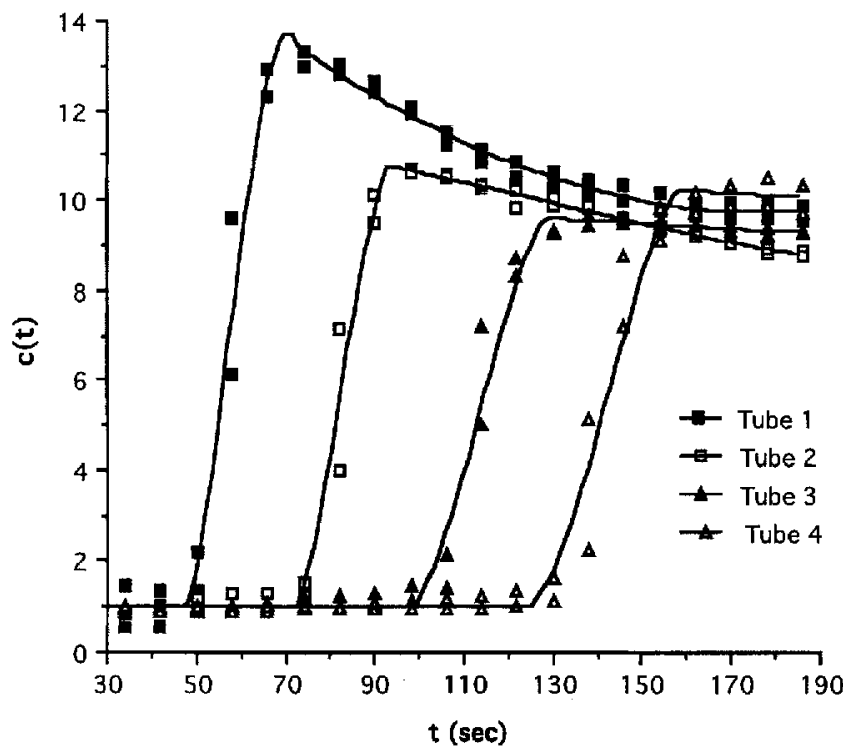

Figure 6. Flow phantom enhancement curves $c(t)$ versus time. At $t=0$, a $1-\mathrm{ml}$ bolus of gadopentetate dimeglumine was administered, followed by a $.42-\mathrm{ml} / \mathrm{min}$ constant infusion.

centric manner. The outermost or "slow" phase encoding was in the $y$ direction. System parameters (frequency, transmit and receive gain) were held constant for all acquisitions. Total scan time was 45 seconds.

Because the phantom was constructed such that each of the four tubes has a different enhancement function $c_{m}(t)$, the effects of four different enhancement curves are evaluated with a single three-dimensional data set. Similar to the simulation study, "imaging time" denotes the time point at which the central line of $\mathbf{k}$ space is acquired. The two imaging times $\left(t_{c k}=74\right.$ and $\left.t_{c k}=90\right)$ were chosen based on the phantom enhancement data (Fig. 6). In particular, these time points bound the period of greatest intensity change in tube 2. At 74 seconds, tube 1 has enhanced maximally and is slowly decreasing in signal intensity, while tube 2 is just beginning rapid enhancement and tubes 3 and 4 have not yet seen gadolinium. At 90 seconds, tubes 1 and 2 have completely enhanced and are slowly decreasing in intensity, while tubes 3 and 4 have not yet enhanced. Both sequential and centric imaging were performed at 74 and 90 seconds after a gadolinium bolus and infusion identical to that described above.

Next, one-dimensional computer modeling was performed using the exact same geometry, timing, and image acquisition parameters as in the phantom images. Centric and sequential modeling data were compared with data generated from a line plot of the 32 centralmost lines within the phantom images (each of the four tubes within the phantom was analyzed separately to ensure that the central slice was evaluated). The modeling data for each run was normalized to tube 1 of the 74 -second centric phantom image.

\section{Image Analysis and Reconstruction}

Vessel mean (Fig. 7) was generated by taking the average intensity along the modeled vessel lumen. Phantom line profiles (Fig. 8) were generated using NIH Image 1.56 (National Institutes of Health, Bethesda, MD).

Illustrative examples of human three-dimensional GdMRA are also presented (see legend of Fig. 9). All patient image analysis and manipulation were performed using a GE Windows workstation (General Electric Medical Sys-

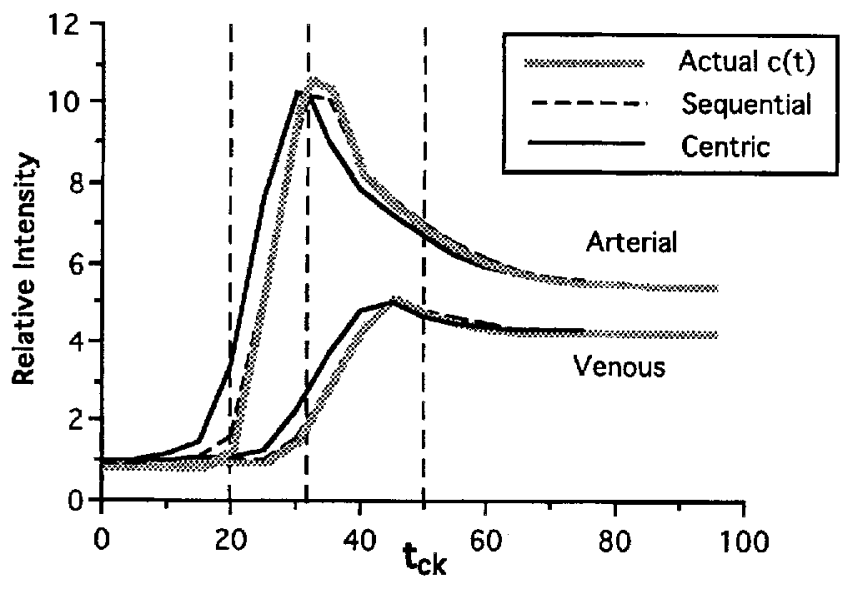

Figure 7. Sequential and centric modeled mean intensity (venous and large arterial "vessels") versus $t$. Plotted versus the "actual" enhancement function $c(t)$ on which the modeling was based. Vertical dashed lines represent the $t_{c k}$ values corresponding to the modeling in Figure 5

tems, Milwaukee, WI). Maximum intensity projections (MIPs) multiplanar relormats and subvolume acquisitions in arbitrary planes were performed.

\section{- RESULTS}

Computer-modeled sequential and centric one-dimensional line profiles are shown in Figure 5 . These are best compared when the center of $k$ space occurs at the same point in time (referred to as $t_{c k}$ ). These data are also plotted as average vessel intensity verses $t_{c k}$ for each technique, which is presented in Figure 7.

Flow phantom Gd-MRA images are seen in Figure 10. The corresponding modeled and measured line profiles are demonstrated in Figure 8. There is close agreement between actual and predicted profiles in terms of gross artifacts, such as strong timing-dependent differential signal intensity, tube-edge "ringing," and significant error in the apparent tube width, especially for the $t_{c k}=74$ seconds data. Although there is some overestimation of signal intensity in tube 1 for $t_{c k}=90$ seconds (more pronounced for the sequential technique, perhaps due to a compromised gadolinium bolus), the relative peak intensities and line profile shapes correlate well over all.

Phantom studies were primarily performed to validate the computational model, thereby allowing us to gain insight into the effects of time-varying intravascular signal on MRA using a fast, simple computer algorithm rather than time-consuming phantom and patient experimentation. Incorrect infusion timing in patients resulted in nondiagnostic images (Figs. $9 \mathrm{~b}$ and $9 \mathrm{c}$ ). Clinical implementation of optimized infusion timing is shown in Figure $9 \mathrm{~d}$. Based on these phantom, model, and clinical results, several important observations can be made regarding time-varying intravascular signal intensity and $\mathrm{k}$ space acquisition order. Each of these is discussed below.

\section{Contrast is Determined at the Center of $k$ Space}

The first and perhaps most important observation is that vessel signal intensity when the center of $k$ space is sampled $\left(t_{c k}\right)$ closely corresponds to the intensity of the corresponding enhancement curve $c(t)$ for both sequential and centric acquisitions (Fig. 7). This makes intuitive sense, because the center of $\mathbf{k}$ space (low spatial frequencies) contributes most to image contrast, whereas the periphery of $\mathrm{k}$ space (high spatial frequencies) contributes more to smaller details and edges (13). From a practical 

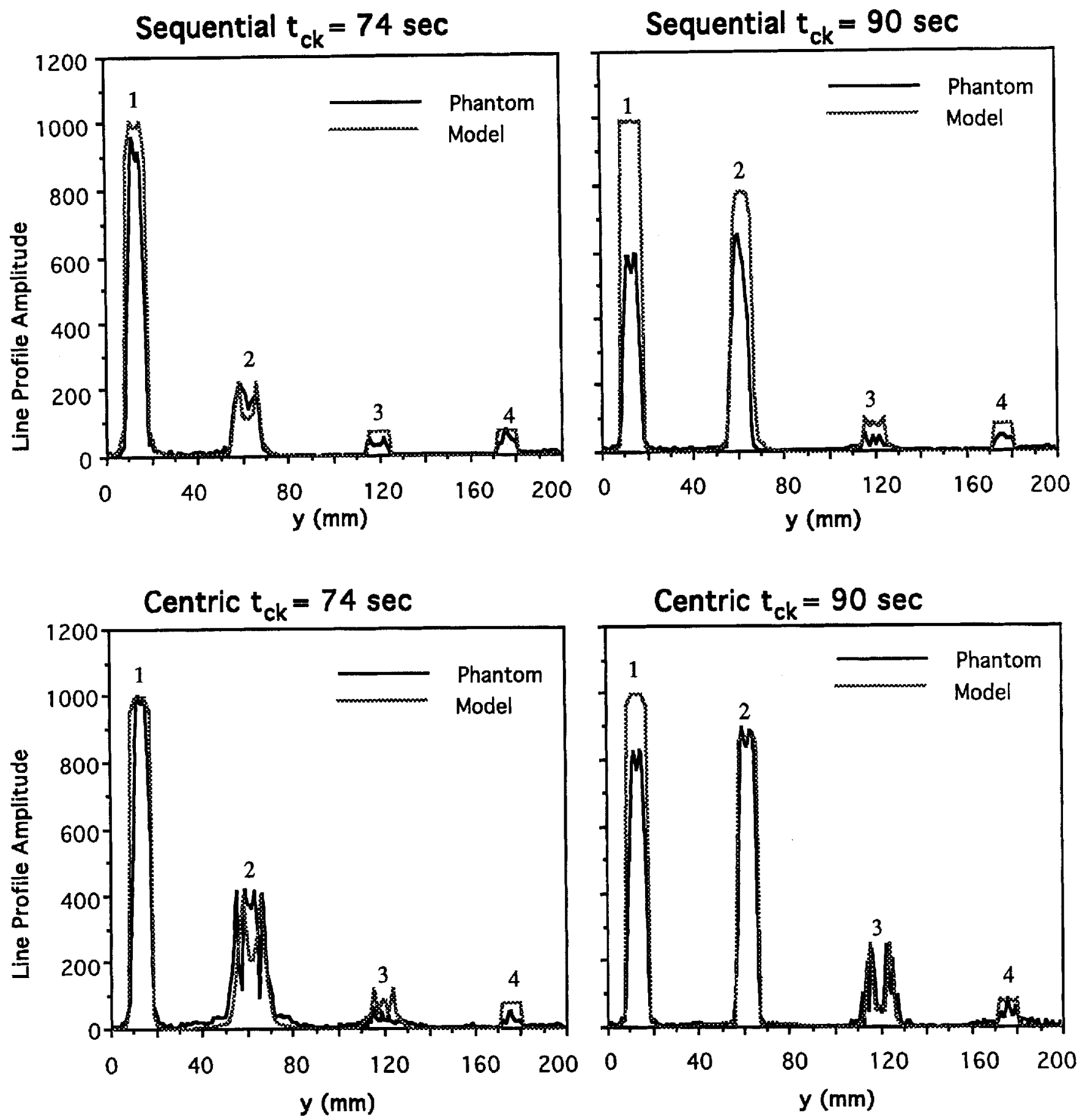

Figure 8. Sequential and centric phantom line profiles versus computer-modeled profiles for $t_{c k}=74$ and 90 seconds. Modeling based on the enhancement data $c(t)$ from Figure 6 . All tubes are approximately $1 \mathrm{~cm}$ in diameter.

standpoint, this means that if an enhancement function $c(t)$ is known, maximal arterial signal intensity can be obtained by choosing the imaging parameters such that the middle of $k$ space is sampled at the time of peak arterial enhancement (termed $t_{p}$ ). Alternatively, the maximum difference in signal between two vessels (such as the aorta and IVC) can be obtained by sampling the middle of $\mathrm{k}$ space when the difference between these two vessels is maximum (see Fig. 4).

A nice example of this is seen in the two left-hand panels of Figures 8 and 10 , in which tube 1 is analogous to an artery and tube 2 is analogous to a vein. Even better differentiation could have been obtained if $t$ were shortened to approximately 70 seconds (to correspond to the peak of tube 1 enhancement). Figure $9 \mathrm{~d}$ demonstrates the potential of clinical MRA using optimum timing parameters. Note the high signal intensity differences between arterial and venous vessels and the lack of significant artifact in this patient with two left renal arteries, both of which have proximal stenoses.

\section{Vessel Size}

Another important observation relates to vessel size (see Fig. 5). For optimal image timing (in this example, $t_{c k}$ $\sim t_{p}=32$ seconds), signal intensity decreases as vessel size decreases. Further simulations demonstrated up to a $25 \%$ maximum signal intensity loss as vessel diameter 


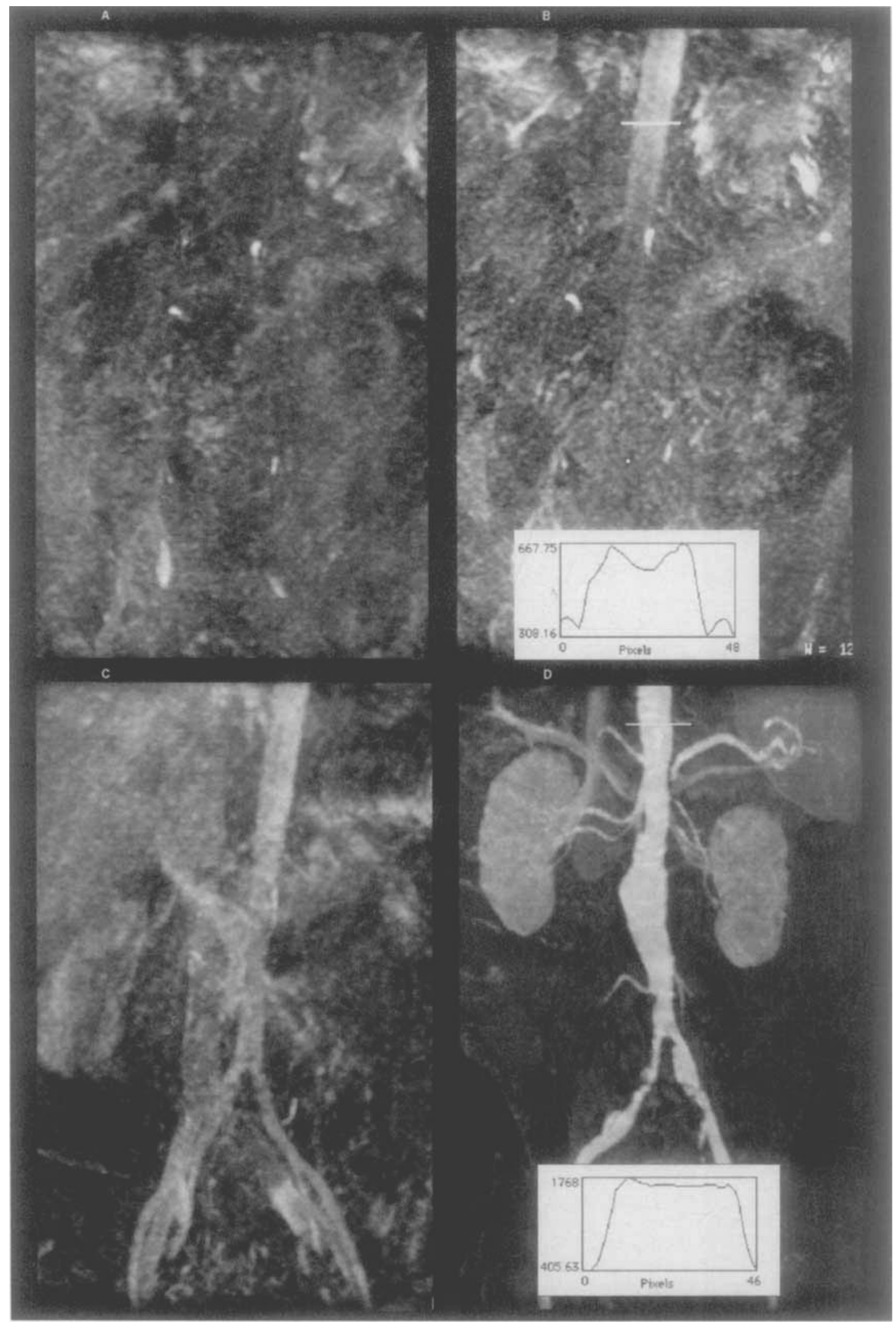

Figure 9. Coronal three-dimensional Gd-MRA MIP images of the abdomen (spoiled gradient echo, 1.5-T GE Signa, TR $=7 \mathrm{msec}$, TE $=2$, flip angle $=45^{\circ}, 256 \times 128, \mathrm{FOV}=32 \mathrm{~cm}, 28$ slices, slice thickness $=2.5 \mathrm{~mm}$, no saturation pulses). (a) pregadolinium, (b) after a $42-\mathrm{ml} \mathrm{N}$ gadopentetate dimeglumine infusion incorrectly timed such that the arterial gadolinium peak occurred after acquisition of central k space, (c) postgadolinium during the equilibrium phase, and (d) timed for peak arterial gadolinium concentration to occur just before central $\mathbf{k}$-space acquisition (different patient than a through $\mathbf{c}$ ). Note that in $\mathbf{b}$, an inset line profile of aortic signal intensity shows the characteristic ringing artifact that occurs when the center of $\mathrm{k}$ space is acquired during rapidly rising arterial gadolinium concentration. A similar line profile in $\mathbf{d}$ is much more uniform. 


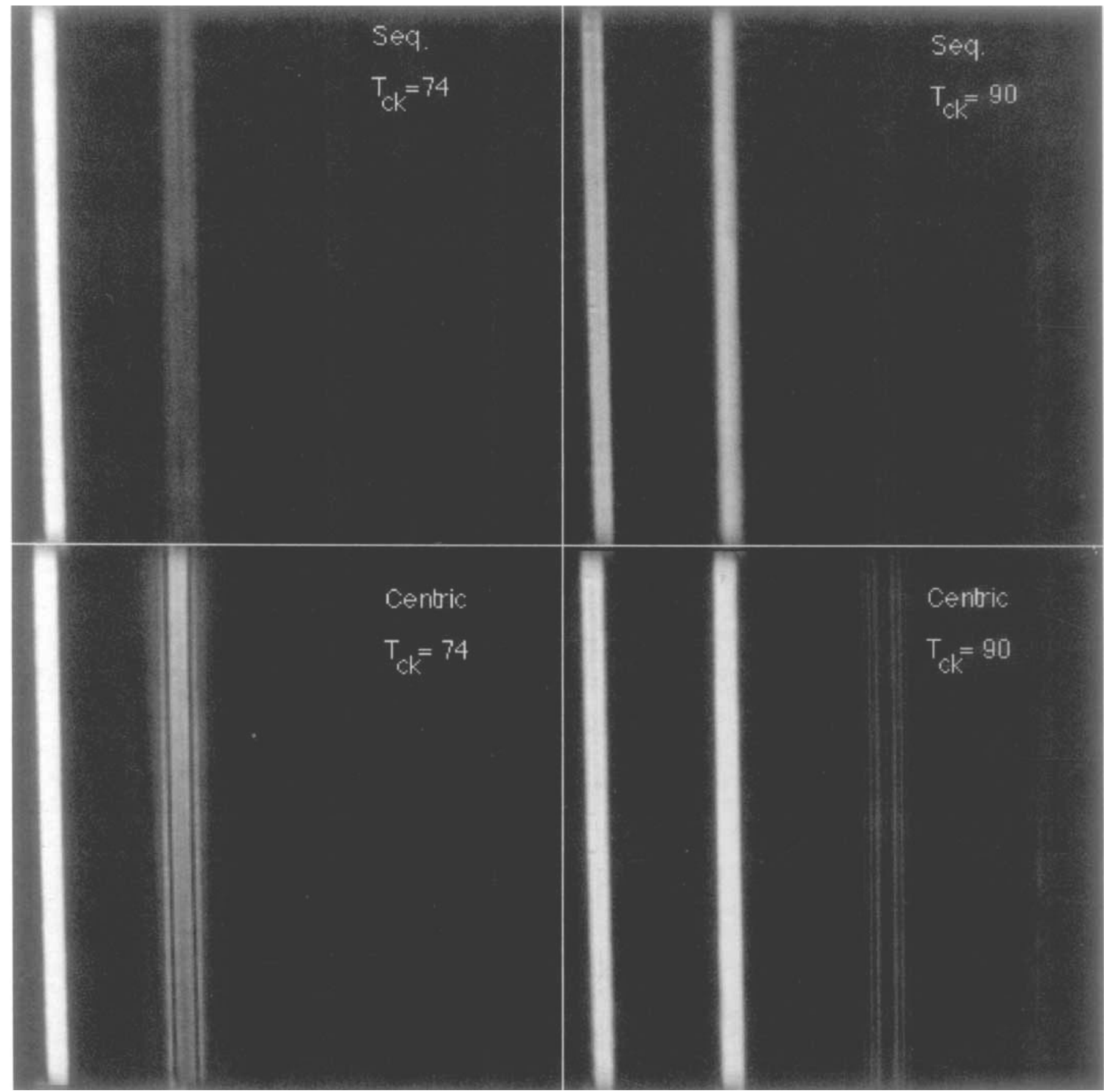

Figure 10. Coronal images through the flow phantom for $t_{c k}=74$ and 90 seconds [sequential and centric). Tube 1 is on the left, tube 4 is on the right.

approached 1 pixel in size. This is no surprise, because the smaller the vessel, the greater the high spatial frequency content, and high frequencies are preferentially attenuated by the filtering function $k\left(k_{y}\right)$ (Fig. $\left.3 b\right)$. This is an important consideration in the attempt to image smaller and smaller vessels using MRA, again stressing the importance of maximizing signal through understanding the dynamics of the gadolinium bolus.

\section{Artifacts}

The artifacts generated by time-varying intravascular gadolinium concentration depend on the way in which the data are collected. Significant line-shape distortion occurs when $t_{c k}<t_{p}$, particularly with centric acquisition. Under these circumstances, there is a coarse "ringing" artifact combined with "widening" of the vessel margins. In addition, with centric acquisition, vessel intensity is overestimated for $t_{c k}<t_{p}$ and underestimated for $t_{c k}<t_{p}$. All of these effects are observed qualitatively in Figures 5 and 8 and quantitatively in Figure 7.

These artifacts can be best understood by considering how the $\mathrm{k}$-space data are modulated by the function $\mathrm{k}\left(k_{\mathrm{y}}\right)$. As shown in Figure $3 a\left(t_{c k}\right.$ significantly earlier than $\left.t_{p}\right)$, both techniques amplify the center of $k$ space significantly less than the higher frequencies, accounting for the "ringing"-type artifacts at the edge of the vessels. The 
centric filter, however, has overall greater magnitude across the central portion of $\mathrm{k}$ space. Hence, there is more area in the Fourier power spectrum, and because total power is conserved between real and Fourier space, extra signal appears in the real image as increased intensity and artifact in the vessel. This means that acquiring data where $t_{c k}$ occurs before $t_{p}$ is particularly disastrous and should be avoided. It also illustrates the importance of understanding the enhancement dynamics $c(t)$ for each vessel imaged. In this manner, maximum signal can be obtained by placing $t_{c k}$ at or near $t_{p}$, and the severe artifacts related to $t_{c k}$ occurring too early can be avoided.

The phantom data (Figs. 8, 10) demonstrate these effects. Comparing the sequential and centric data for $t_{c k}=$ 74 seconds, note that tube 1 is maximally enhanced and relatively artifact-free for both techniques, as expected. Tube 2 demonstrates increased amplitude, broadening, and a "ringing"-type artifact spread out in the phase-encoding direction $(y)$ as predicted for toccurring before or during the rapid upslope of $\kappa\left(k_{1}\right)$. Note also that as predicted, the effects of $t_{c k}$ occurring too early are more severe for centric acquisition (see, in particular, tube 3 of the $t_{c k}=90$ seconds data). This ringing artifact also was observed in patients when the infusion was not timed properly (see the inset line profile across the aorta in Figs. $9 b$ and $9 d)$.

\section{- DISCUSSION}

For most MR imaging, tissue signal intensity remains constant during image acquisition. Even after gadolinium administration, scan times are short compared to the elimination time for renal excretion. However, an increasing number of studies are being performed "dynamically," that is, during and immediately after contrast infusion. Under these circumstances, the effects of time-varying gadolinium concentration must be considered. The theoretical modeling, phantom studies, and patient examples presented here demonstrate some of the useful effects and artifacts caused by the time-varying signal intensity during image acquisition.

Based on this work, several practical considerations for Gd-MRA become evident. Most importantly, the signal intensity of the vascular structure of interest (or differences between vessels of interest) can be manipulated by knowing the time course of the intravascular gadolinium concentration and acquiring the image with the center of $k$ space at the desired point in the enhancement curve $c(t)$. If the timing is chosen such that $t_{c k} \sim$ arterial $t_{p}$, the arterial artifacts are minimal, and arterial enhancement greatly surpasses venous enhancement. As seen in Figures 4 and 7 , however, the window of opportunity for separating arterial and venous structures in this manner is limited, as arterial enhancement peaks sharply with only an approximately 15-second delay between peak arterial and venous enhancement. Hence, knowing or accurately predicting the dynamics of intravascular enhancement after contrast agent administration is extremely important. Such dynamics have been investigated, not only in the MR literature but also in the literature on computed tomography and digital subtraction angiography $(12,14-$ 17). According to data from Strouse et al (12), peak aortic enhancement time after a 10-second gadolinium bolus was somewhat variable, ranging from 29 to 42 seconds (average of 34 seconds with a standard deviation of 5.5 seconds). Such patient-to-patient variability is a fundamental concern, as accurate de novo prediction of vascular enhancement is difficult.

If the center of $\mathrm{k}$ space is inadvertently acquired too early $\left(t_{c k}<t_{p}\right)$, scvere artifacts are generated, particularly with centric acquisition. These artifacts consist mainly of ringing and widening of the apparent lumen in the slow phase-encoding direction. In clinical imaging, artifacts such as these can lead to overestimation of vessel diameter as well as a linear banding pattern in the vessel (which may be mistaken for ghosting secondary to motion). These effects will certainly hinder diagnostic use. In addition, despite having the same gadolinium concentration as adjacent larger vessels, small (or stenotic) vessels have decreased magnitude, even with optimum timing of $t_{c k}$. This will limit the use of Gd-MRA in extremely small vessels.

Considering the somewhat less forgiving artifact characteristics of the centric technique, why consider it at all? Centric acquisition offers a significant advantage when used in conjunction with breath-holding. Preliminary work suggests the most critical period for breath-holding is through the central portion of $k$ space. It is much simpler to have patients breath-hold from the beginning of a centric acquisition than to instruct them to breath-hold in the middle of a sequential acquisition, especially because it is difficult to predict how long the breath-hold will last or if they will hear the instructions over the noise of the gradients.

Total acquisition time is yet another variable to consider when discussing artifacts generated by time-varying intravascular signal intensity. The shorter the data acquisition, the more constant the "filter" function $c(t)$ becomes, with a resultant decrease in vessel artifact. Ideally, an instantaneous "snapshot" image (such as echoplanar imaging) could be used, with total elimination of all artifacts related to time-varying intravascular signal intensity. This would have the added benefit of eliminating motion artifacts but the disadvantage of a lower signal-to-noise ratio.

\section{- CONCLUSION}

Using Gd-MRA, maximum arterial signal intensity with a minimum of artifact occurs when the infusion is timed such that the acquisition of the central portion of $\mathrm{k}$ space coincides with peak arterial gadolinium concentration.

\section{References}

1. Creasy J, Price R, Presbrey T, Goins D, Partain C, Kessler R. Gadolinium-enhanced MR Angiography. Radiology 1990; 175:280-283.

2. Prince M, Yucel E, Kaufman J, Harrison D, Geller S. Dynamic gadolinium-enhanced 3DFT abdominal MR arteriography. J Magn Reson Imaging 1993; 3:877-881.

3. Prince M. Gadolinium-enhanced MR aortography. Radiology 1994; 191:155-164.

4. Kaufman J, Geller S, Petersen M, Cambria R, Prince M, Waltman A. MR imaging (including MR angiography) of abdominal aortic aneurysms: comparison with conventional angiography. AJR 1994; 163:203-210.

5. Snidow J, Aisen A, Harris V, et al, Iliac artery MR angiography: comparison of three-dimensional gadolinium-enhanced and two-dimensional time-of-flight techniques. Radiology 1995; 96: 371-378.

6. Marchal G, Bosmans J, Van Hecke P, Jiang Y, Aerts P, Bauer H. Experimental Gd-DTPA polylysine enhanced MR angiography; sequence optimization. J Comput Assist Tomogr 1991; 15(4):711-715

7. Marchal G, Bosmans H, McLachlan S. Magnetopharmaceuticals as contrast agents. In: Potchen EJ, Haacke EM, Siebert JE, Gottschalk A, eds. Magnetic resonance angiography: concepts and application. St. Louis: Mosby, 1993; 305-311.

8. Prince M, Narasimham D, Stanley J, et al. Gadolinium-en hanced magnetic resonance angiography of abdominal aortic aneurysms. J Vasc Surg 1995; 21:656-669.

9. Holsinger A, Riederer $\mathrm{S}$. The importance of phase encoding order in ultra-short TR snapshot MR imaging. Magn Reson Med $1990 ; 16: 481-488$. 
10. Jones R, Rinck R. Approach to equilibrium in snapshot imaging. Magn Reson Med 1990; 8:797-803.

11. Prince M, Narasimham D, Stanley J, et al. Breath-hold gadolinium-enhanced MR angiography of the abdominal aorta and its major branches. Radiology 1995; 197:785-792.

12. Strouse $P$, Prince M, Chenevert T. Dynamic vascular and soft tissue enhancement patterns in abdominal MRI. In: Proceedings of the 3rd scientific meeting of the Society of Magnetic Resonance. Nice, France: Society of Magnetic Resonance, 1995; 533.

13. Riederer S, Tasciyan T, Farzaneh F. MR fluoroscopy: technical feasibility. Magn Reson Med 1988; 8:1-15.
14. Claussen C, Banzer D, Pfretzschner C, Kalender W, Schorner W. Bolus geometry and dynamics after intravenous contrast medium injection. Radiology 1984; 153:365-368.

15. Rubin G, Dake M, Napal S, et al. Spiral CT of renal artery stenosis: comparison of three-dimensional rendering techniques. Radiology 1994; 190:181-189.

16. Becker $\mathrm{G}$. Holden R. Sodium dehydrocholate circulation times in digital subtraction anglography. AJR 1983; 140:817-818.

17. Mirowitz S, Gulierrez E, Lee J, Brown J, Heiken J. Normal abdominal enhancement patterns with dynamic gadolinium-enhanced MR imaging. Radiology 1991; 180:637-640. 